\title{
Study of Vertebral Body Replacement with Reconstruction Spinal Cages in Dorsolumbar Traumatic and Koch's Spine
}

\author{
Rohit Anilbhai Thaker ${ }^{1}$, Vinod K Gautam ${ }^{2}$ \\ ${ }^{1}$ Department of Orthopedics, K.J. Somaiya Medical College, Mumbai, India \\ ${ }^{2}$ Department of Orthopaedics, B.J.Medical College, Civil Hospital, Ahmedabad, India
}

Study Design: Retrospective and prospective case series.

Purpose: The aim of this study was to assess the results of reconstruction of anterior column, fusion and complications related to cages.

Overview of Literature: Literature shows that corpectomy has become a common surgical procedure for spinal infection, trauma, deformity, instability and metastasis. Also the use of reconstructive spinal cages is common after corpectomy.

Methods: Study was carried out in patients with dorsolumbar traumatic and Koch's spine. We assessed 25 patients (13 traumatic/12 tuberculous) who were treated with cages with/without any other instrumentation. Radiographs were obtained before and after the surgery. A preoperative magnetic resonance imaging was obtained in every patient.

Results: Fourteen patients underwent 1 level of corpectomy, 9 patients underwent 2 levels and 2 patients underwent 3 levels of corpectomy. Anterior reconstruction alone was performed in 8 patients; $360^{\circ}$ reconstruction was performed in 17 patients and 2 of them underwent reconstruction through single posterior approach only. The mean kyphotic angulation improved from $21.2^{\circ}$ preoperatively to $9.3^{\circ}$ postoperatively and to $12^{\circ}$ at final follow up. 8 patients with neurological deficits had improvement by at least one or more Frankel grade. No migration/displacement of cage was seen in any patients.

Conclusions: The present study demonstrates that the vertebral body replacement after corpectomy by reconstructive cages provides a reconstruction of the anterior column, good correction of the mean kyphotic angle, and a correction maintained with cage without any cage related complication at long term follow up. The fusion can be achieved with reconstructive cage plus bone graft with or without posterior instrumentation.

Keywords: Spinal fusion; Reconstructive surgical procedure; Autografts; Spinal Injuries; Tuberculosis, spinal; Tuberculosis, multi drug resistant

\section{Introduction}

Corpectomy has become a common surgical procedure for spinal infection, trauma, metastasis, deformity, in- stability and degenerative stenosis $[1,2]$. Autogenous bone grafts such as iliac crest or fibula have been used to reconstruct the anterior column despite of donor site morbidity and pseudarthrosis and graft dislodgement are

Received Dec 6, 2013; Revised Mar 9, 2014; Accepted Apr 23, 2014

Corresponding author:Rohit Anilbhai Thaker

Department of Orthopedics, K.J. Somaiya Medical College, Room No.6, RMO hostel,

4th floor, Everard Nagar, Sion, Mumbai-400022, India

Tel: +91-22-24020933, Fax: +91-97-02720969, E-mail: thakerrohit1@gmail.com 
well known complications [3]. Ideally, a vertebral body replacement system should be stable and resist axial loadbearing. Also it should have a large interbody-bone interface to facilitate fusion, prevent migration and restore height and sagittal alignment [4].

Most thoracolumbar burst fractures are stable injuries that can be treated nonoperatively [5]. However, unstable burst fractures resulting in neurologic deficits with retro pulsed bone fragments and canal compromise or burst fractures with disruption of the posterior osteoligamentous complex usually are unstable injuries that warrant surgical intervention. The ideal treatment for unstable thoracolumbar burst fractures is controversial but the reconstruction of all three columns of Denis spine model is necessary in case of a severe traumatic spinal injury.

Tuberculosis of the dorsolumbar spine is very common in the Indian population. The frequency of the disease is closely related to the patients' social, economical and education status [6]. The majority of patients with Koch's spine with an initial stage of disease can be treated by an anti- tubercular treatment only [7]. But abscess formation and destruction of the vertebral body occur if the disease is advanced. This produces kyphotic deformity and canal compromise with cord compression. Abscess drainage, the removal of the diseased vertebral body and debris and decompression of the spinal cord is necessary in such cases. The aims of treatment are to eradicate the disease and to prevent the development of paraplegia and kyphotic deformity. And if it exists, the management of the same may also be an aim of treatment [8].

The spinal cage has been widely used in anterior decompression and reconstruction in cases of Koch's spine [9]. Also it has been widely used for anterior column reconstruction in cases of traumatic spine [10]. It promotes intervertebral fusion and achieves a high fusion rate if it is combined with bone graft. Varieties of spinal cages are available for various purposes and continuous advancement is being made to improve the design and material of the cage to promote an easier insertion technique and provide sufficient anterior stabilization. Expandable and non-expandable reconstruction interspinal cages are used in the treatment of dorsolumbar traumatic and Koch's spine, where vertebral body replacement is needed after total or partial corpectomy of one or more vertebral bodies and intervertebral discs.

A circumferential anterior as well as posterior column reconstruction is provided if the spinal cage is combined with posterior instrumentation. More kyphotic correction can be achieved and maintained with the cage than with the posterior instrumentation alone. The cage is usually inserted through anterior approach which eases the removal of any retro pulse fragment, abscess or granulation tissue under vision. This action results in a decompression of the cord which aids to neurological improvement.

\section{Materials and Methods}

Thirteen patients with traumatic dorsolumbar spine and twelve patients with Koch's spine were treated with corpectomy and reconstructive spinal cage between 2009 to 2011, at our institute and were retrospectively and prospectively studied.

The age of these patients ranged from 12 to 60 years with a mean age of 32 years. There were 14 males and 11 females patients. Nine patients with lumbar level involvement while 14 patients were of dorsal level involvement and 2 patients had a dorsolumbar junction involvement. Of patients with traumatic spine, were six patients having other associated injuries along with the spinal trauma. Eight patients were only treated with anterior decompression and cage plus bone graft, while 17 patients were treated with anterior plus posterior stabilization in the form of pedicle screw or rectangular frame with sub laminar wire.

The material of all cages used in the present study series was stainless steel, 8 patients were treated with expandable cage design and 17 were treated with simple nonexpandable mesh cage. All cages were having a round grid pattern and spikes at both ends of the cage. No reinforcement ring was used in any of the patients.

All patients were preoperatively assessed in form of clinical examination and radiological investigation. Demographic data were collected of all patients and the functional outcomes were determined on the basis of Denis pain scale and work scale. The grading for Neurological improvement was taken from multicenter spine fracture study conducted by Scoliosis Research Society coordinated by Gertzbein [11]. Preoperative radiographs and magnet resonance imaging (MRI) of involved diseased vertebral levels were done in all patients. Postoperative $\mathrm{X}$-rays were obtained in all patients. X-rays were done at final follow up to assess fusion and status of the cage. Also computed tomography scans were done in some patients. 
Table 1. Bridwell grading for fusion [13]

\begin{tabular}{llc} 
Fusion grade & Description & Points \\
\hline Grade I (definite) & Fused with remodelling of trabeculae & 5 \\
Grade II (probable) & Graft intact but not fully remodelled or incorporated. No lucency & 3 \\
Grade III (probably not) & Graft intact; definite lucency present at graft host junction & 1 \\
Grade IV (not fused) & Bone graft resorption with collapse; cage graft settling down or angulatedMithLucency & 0 \\
\hline Grade V & Inability to assess especially in case of radiopaque cage graft & - \\
\hline
\end{tabular}

The segmental kyphosis was determined by measuring the Cobb's angle. All patients were neurological assessed according to the modified Frankel grading preoperatively and at final follow up [12]. The outcome data were collected preoperatively, postoperatively, at discharge and at final follow up. The minimum follow up duration was nine months and fifteen months on average. At final follow up, the fusion was determined by Bridwell grading system for fusion (Table 1) [13].

\section{Surgical techniques}

In our present study were the approaches used for anterior surgery as follows: 1) For dorsal spine D2-D5: left periscapular or transpleural approach. 2) For dorsal spine D6 to D11: left transpleural approach. 3) For D-L junction DI2-L1: left transdiaphragmatic approach, opening the Pleural and retroperitoneal space. 4) For lumbar spine L2 to LS: left retroperitoneal approach.

Decompression of the cord was achieved by removing fractured or diseased body leaving the anterior and right lateral cortex intact as much as possible. The intervertebral discs above and below the level are removed to expose the adjacent end plates which were partially curetted to bleed. Sagittal slots were made in the adjacent vertebrae to put cage with graft in the slots if end pates were damaged. Cages of proper diameters and lengths were inserted to gain better hold. Graft or cage was anteriorly placed at a maximum distance to the spinal canal but not beyond the premises of the vertebral body. In this study, anterior Moss-Miami and $\mathrm{Z}$ plate-anterior thoracolumbar construct system were used for anterior instrumentation when required. Screws were fixed with Rod/Z-ATL plate system and locked. The screws were of $5 \mathrm{~mm}$ diameter and bicortically inserted with good purchase. This anterior instrumentation was used in trauma only and not in diseased spine. No over compression was tried beyond the full contact of cage or graft with intact vertebrae. In case of posterior surgery were patients placed in prone position over the spinal frame (Relton Hall frame) or on the bolsters with free abdomen and hips and knees in some flexion. Exposure was done by a standard midline approach. The levels were confirmed by peroperative Xrays. The Roy Camille method or more specifically the Magerl method modified by Krag [14] was used for lumbar spine and the Cinotti et al. [15] method was used for thoracic spine to search the entry point of pedicle screws. In two patients the cage with bone graft was anteriorly placed by posterolateral approach through a single posterior midline incision. Decortications of the facets and lamina were performed to facilitate fusion. Local bone graft was placed to facilitate a posterolateral fusion.

\section{Results}

The study group consisted of 14 male and 11 female patients with a mean age of 32 years (range, 12 to 60 years). The mean duration of follow up was 15 months (range, 9 months to 36 months). 13 patients had a traumatic injury and 12 patients presented with Koch's spine. Of the 13 patients with traumatic DL spine injury were 3 injured by a road traffic accident and the rest of them had an injury due to a fall from height. Of the 12 patients with dorsolumbar Koch's spine had 8 patients a history of BCG vaccination and 2 patients had a history of pulmonary Koch's. All traumatic spine patients presented within 24 to 48 hours after accident and were attended immediately. All efforts were made to operate these patients as early as possible. The average injury surgery interval was 7 days (range, 2 to 30 days) and the average interval between anterior and posterior surgery was 14 days (range, 7 to 30 days). Every patient received blood transfusion. The mean hospital stay was 18 days (range, 10 to 30 days).

Fourteen patients underwent a single level corpectomy, 
nine patients underwent a double level and two patients underwent a three level corpectomy. Anterior reconstruction alone was performed in eight patients; $360^{\circ}$ reconstruction was performed in seventeen patients and two of them underwent the reconstruction through posterior approach only.

The mean kyphotic angulation improved from $21.2^{\circ}$ preoperatively to $9.3^{\circ}$ postoperatively and increased to $12^{\circ}$ during an average follow-up duration of 15 months. Grade I fusion was achieved in $21(84 \%)$ patients. Grade II fusion was achieved in 3 and grade IV fusion was evident in 1 patient. No migration or displacement of cage was seen in any patients, but subsidence was seen in eleven patients. It was higher in Koch's spine patients (8 out of 12) than in traumatic spine patients (3 out of 13). Subsidence was with 4 out of 8 (50\%) little higher in patients with anterior reconstruction alone, while it was 7 out of $17(41 \%)$ in patients with $360^{\circ}$ reconstruction. No reinforcement ring to prevent subsidence was used in the present study.

At presentation, neurological deficits were present in 20 patients. Eight patients with neurological deficits had improvement by at least one or more Frankel grade. One patient showed neurological worsening postoperatively but recovered at final follow up while one other patient showed neurological worsening of one Frankel grade. The cause of permanent neurological deterioration in the latter patient could have been due to ischemic damage to the cord because of disease progression secondary to failure of response to treatment. All the patients with normal neurology remained same till final follow up (Table 2). Implant failure occurred in two patients and the pedicle screw cut out through the vertebral body with minimal cage migration in both of these patients. No cage related complications were noted like cage displacement or penetration in viscera or spinal canal. 21 patients showed a central placement of cage on anteroposterior and lateral radiograph, while 4 patients showed a paracentral placement. There were no significant changes in cage placement till the latest follow up.

Every effort was made to mobilize the patients as early as possible after surgery 6 patients were walking without any support at their latest follow up and 9 patients were walking with support. 9 patients achieved wheel chair activity. 1 patient remained bed ridden till final follow- up and the same patient was found to have non-union and neurological worsening because of an advancement of
Table 2. Improvement in Frankel grading

\begin{tabular}{|c|c|c|c|c|c|c|}
\hline \multirow{2}{*}{\multicolumn{2}{|c|}{$\begin{array}{c}\text { Frankel grading } \\
\text { Preoperative }\end{array}$}} & \multicolumn{5}{|c|}{ Postoperative } \\
\hline & & A & B & C & D & $E$ \\
\hline A & 8 & 5 & 1 & 2 & & \\
\hline B & 3 & & 1 & 1 & 1 & \\
\hline C & 5 & & 1 & 1 & 3 & \\
\hline D & 4 & & & & 4 & \\
\hline E & 5 & & & & & 5 \\
\hline
\end{tabular}

its disease process. Later, this patient was found to have multi drug resistant tuberculosis. An antitubercular treatment was started according to drug sensitivity but the patient succumbed to disease process because of widespread dissemination of disease at 2 years follow up. No attempt of any revision surgery for non-union was done in this patient.

A superficial infection was noted in three patients and was treated with appropriate antibiotics. Bed sore was noted in 7 patients, urinary tract infection in 2 patients and chest infection in 1 patient during hospital stay. All these complications were early attended and appropriately treated.

Grading for Neurological improvement was taken from multicenter spine fracture study conducted by Scoliosis Research Society coordinated by Gertzbein. Final results were calculated by Denis pain (Fig. 1) and work scale (Fig. 2), Scoliosis Research Society score for neurology and Bridwell grading score for fusion. Combined score of these parameters were taken for outcome measures. Excellent (total score, 16-20) results were obtained in seven (28\%) patients, good (total score, 11-15) results were obtained in ten (40\%) patients; fair (total score, 6-10) results were obtained in five $(20 \%)$ while poor (total score $<6$ ) results were obtained in three (12\%) patients (Figs. 3, 4).

\section{Discussion}

The removal of one or more vertebral bodies followed by spinal column reconstruction continues to evolve. Traditionally, the implantation of autologous tricortical iliac bone graft was the "gold standard" to reconstruct the corpectomy defect. Autogenous bone grafts such as iliac crest or fibula have been used to reconstruct the anterior column, but donor site morbidity, pseudarthrosis and graft dislodgement are well known complications [3]. In 
case of traumatic spine, the corpectomized body can be used as an autogenous graft, while a rib can be used as autogenous bone graft in anterior approach which reduces the donor site morbidity. The correct positioning of non-expandable cages can be challenging. Some distraction forces and the shaping of the endplates may be necessary to insert a rigid implant. Even then, the position of the implant can be improper; endplates can weaken with consequent subsidence and the restoring of the sagittal alignment can be insufficient. An easy, non-distracted insertion of the cage is one of the main advantages of expandable cages for vertebral body replacement. The height can be adjusted to the corpectomy defect in situ and a correction of deformity and restoring height can be achieved. In the present study, the vertebral body replacement was done with $12^{\circ}$ mean kyphotic angle correction by the use of both, expandable and non-expandable cages. The kyphotic correction lost was only $3^{\circ}$ during an average follow up of nine months. It suggests that the anterior column reconstruction with cage is sufficient to provide enough stability for a prevention of a further progression of the kyphotic deformity.

Subsidence was seen in almost half patients in the present study; it may be due to diseased end plates where the

No. of patients

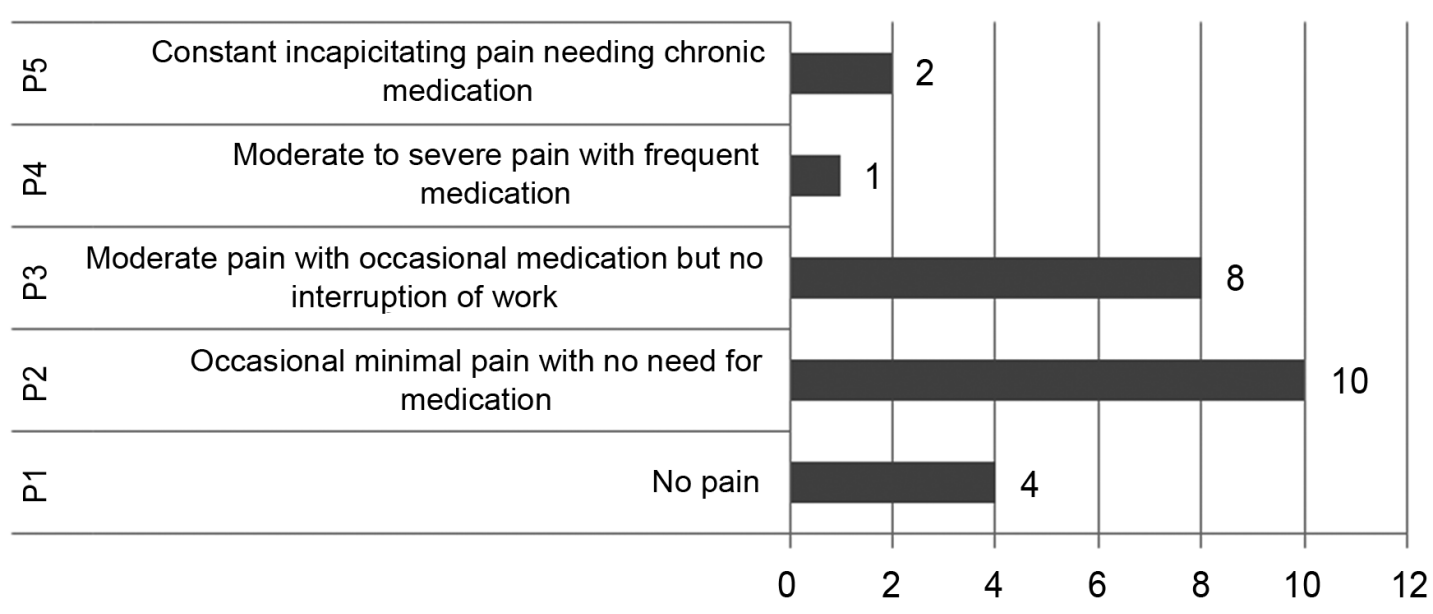

Fig. 1. Denis pain scale.

No. of patients

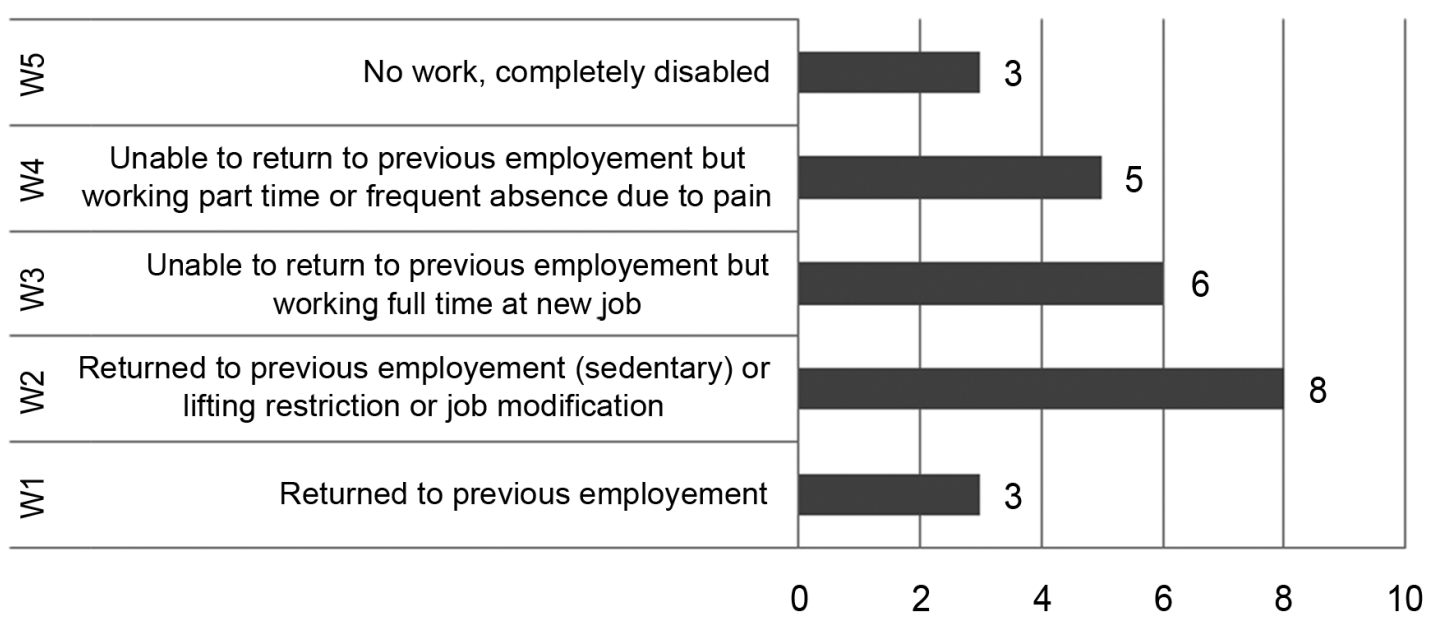

Fig. 2. Denis work scale. 
cage rests or may be due to improper end plate preparation. The fusion rate was good in the present study. All patients were treated with cages filled with bone graft. The central canal of cage was sufficient to allow growth of new bone. Consolidation around the implants was visible and no signs of instability were noted.

The complication rate was low in our study. No significant displacement or migration of cage was noted except in 2 cases where the posterior instrumentation failed. But in those cases the cages were in confinement of the verte- bral body. It may be due to a spike at the ends of the cage which got good hold in the end plates. Expandable cages were relatively easy to insert compared to non-expandable cages. In the thoracic spine, a wide unilateral costotransversectomy allowed an extracavitary decompression of the dura across the midline to the contralateral pedicle through the posterolateral approach. Depending on the pathology, resection of the vertebral body was achieved unilaterally or bilaterally, which obviated the risks associated with a transcavitary approach. However, a rhizotomy
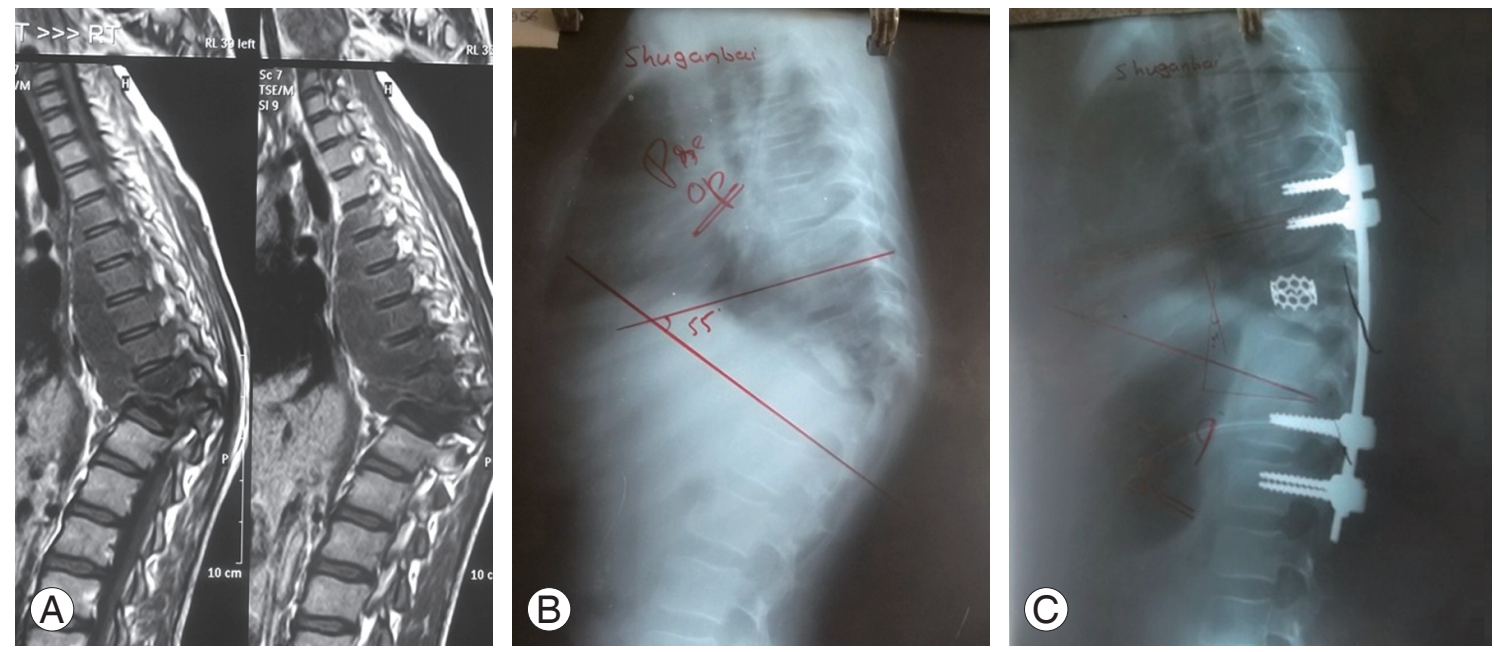

Fig. 3. (A) T1-weighted sagittal images of magnetic resonance imaging of a 12-year-old girl suffering from D10-D11 Koch's spine. (B) Preoperative lateral X-ray of a 12-year-old girl suffering from D10-D11 Koch's spine showing a preoperative kyphotic angle of 55 degree. (C) Postoperative lateral X-ray of a 12-year-old girl suffering from D10-D11 Koch's spine, treated with a $360^{\circ}$ vertebral body reconstruction from posterior approach only, using pedicular screw and a nonexpendable mesh cage.
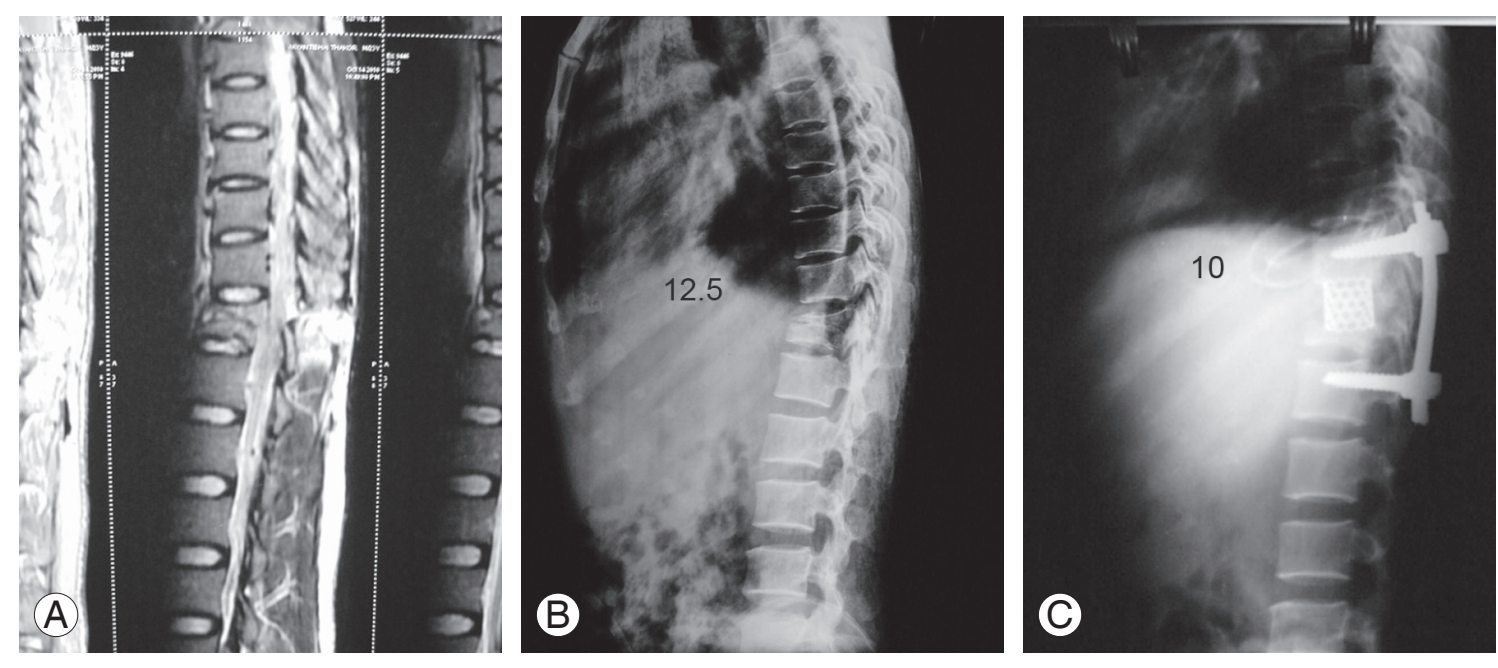

Fig. 4. (A) T2-weighted sagittal image of magnetic resonance imaging of a 40-year-old male having traumatic D12 wedging with complete paraplegia. Preoperative (B) lateral X-ray of a 40-year-old male with traumatic D12 wedging with complete paraplegia, showing a preoperative kyphotic angle of 12.5 degree. (C) Postoperative lateral X-ray of a 40 -year-old male with traumatic D12 wedging with complete paraplegia, treated with posterior pedicle screw fixation followed by anterior vertebral body replacement using a non expandable vertebral mesh cage. 
of 1 segmental nerve root could be necessary to insert the smallest nondistracted expandable cage. Neurological deficits after rhizotomy of a single spinal thoracic nerve root are usually limited due to an extensive overlap of distributions. In cases of lumbar spine involvement, the implant can be horizontally inserted and then vertically rotated when in position. This may allow an insertion of the implant without sacrificing the nerve root.

\section{Conclusions}

In the present study, it can be concluded that vertebral body replacement after corpectomy by using reconstructive cages (expandable as well as non-expandable) provides 1) a reconstruction of the anterior column, 2) a good correction of the mean kyphotic angle, 3 ) a correction was maintained with the cage without any cage related complications at long term follow up. A subsidence rate was noted in almost half of the patients and it was the matter of concern. A good fusion rate can be achieved with a reconstructive cage plus bone graft with or without posterior instrumentation in Koch's spine where posterior elements are intact. A $360^{\circ}$ reconstruction is preferred in a traumatic spine where the posterior instability is a matter of concern. The limitation of the present study was the short duration of follow up (15 months); so, long term results cannot be concluded upon this.

\section{Conflict of Interest}

No potential conflict of interest relevant to this article was reported.

\section{References}

1. Alleyne CH Jr, Rodts GE Jr, Haid RW. Corpectomy and stabilization with methylmethacrylate in patients with metastatic disease of the spine: a technical note. J Spinal Disord 1995;8:439-43.

2. McDonough PW, Davis R, Tribus C, Zdeblick TA. The management of acute thoracolumbar burst fractures with anterior corpectomy and Z-plate fixation. Spine (Phila Pa 1976) 2004;29:1901-8.

3. Schnee CL, Freese A, Weil RJ, Marcotte PJ. Analysis of harvest morbidity and radiographic outcome using autograft for anterior cervical fusion. Spine (Phila Pa 1976) 1997;22:2222-7.
4. Benzel EC. Biomechanics of spine stabilization. New York: Thieme Medical Publishers; 2001.

5. Wood K, Buttermann G, Mehbod A, Garvey T, Jhanjee R, Sechriest V. Operative compared with nonoperative treatment of a thoracolumbar burst fracture without neurological deficit. A prospective, randomized study. J Bone Joint Surg Am 2003;85:773-81.

6. Janssens JP, de Haller R. Spinal tuberculosis in a developed country. A review of 26 cases with special emphasis on abscesses and neurologic complications. Clin Orthop Relat Res 1990;(5):67-75.

7. Moon MS, Moon YW, Moon JL, Kim SS, Sun DH. Conservative treatment of tuberculosis of the lumbar and lumbosacral spine. Clin Orthop Relat Res 2002;(398):40-9.

8. Moon MS. Tuberculosis of the spine. Controversies and a new challenge. Spine (Phila Pa 1976) 1997;22:1791-7.

9. Garg B, Kandwal P, Nagaraja UB, Goswami A, Jayaswal A. Anterior versus posterior procedure for surgical treatment of thoracolumbar tuberculosis: A retrospective analysis. Indian J Orthop 2012;46:165-70.

10. Hunt T, Shen FH, Arlet V. Expandable cage placement via a posterolateral approach in lumbar spine reconstructions. Technical note. J Neurosurg Spine 2006;5:271-4.

11. Gertzbein SD. Scoliosis Research Society. Multicenter spine fracture study. Spine (Phila Pa 1976) 1992;17:528-40.

12. Frankel HL, Hancock DO, Hyslop G, et al. The value of postural reduction in the initial management of closed injuries of the spine with paraplegia and tetraplegia. I. Paraplegia 1969;7:179-92.

13. Bridwell KH, Lenke LG, McEnery KW, Baldus C, Blanke K. Anterior fresh frozen structural allografts in the thoracic and lumbar spine. Do they work if combined with posterior fusion and instrumentation in adult patients with kyphosis or anterior column defects? Spine (Phila Pa 1976) 1995;20:1410-8.

14. Krag MH. Biomechanics of thoracolumbar spinal fixation. A review. Spine (Phila Pa 1976) 1991;16:S8499.

15. Cinotti G, Gumina S, Ripani M, Postacchini F. Pedicle instrumentation in the thoracic spine. A morphometric and cadaveric study for placement of screws. Spine (Phila Pa 1976) 1999;24:114-9. 